\title{
Reward size effects in the double runway'
}

\author{
LAWRENCE DACHOWSKI \\ TULANE UNIVERSITY, NEW ORLEANS
}

Rats received one of three sizes of reward or no reward in the first goal box of a double runway. Reward size had some effect on Runway-1 running and goal speeds, but never-rewarded $S$ s did give acquisition curves for these measures and start scores. The only Runway-2 effect was a temporary depression of start speed for Ss given the smallest reward. Openfield pretesting did not affect speeds.

A double runway has been used to assess the energizing property of frustrative nonreward (Amsel, 1958; Amsel \& Roussel, 1952). The goal box (G1) of the first runway (R1) is the start box of the second runway (R2), so that when partial reinforcement is given in G1 the frustration drive on nonrewarded trials will increase the speed of running in R2. The frustration interpretation depends on demonstrating that the reward given in G1 does not depress speed of running in R2 below the speed of a group which is never rewarded in G1 (Wagner, 1959; Hamm, 1967).

In the present study three sizes of reward in G1 were used in an attempt to determine the maximum reward size which would not depress $\mathrm{R} 2$ responses below the level of a never-rewarded control group. These four levels were combined factorially with two conditions (Before or After runway training) of open-field tests, the results of which are not germane to this paper. Method

The Ss were 71 male albino rats, 115 days old. All Ss were placed on a 22-1/2 h food deprivation schedule and maintained in reversed dark-light eonditions for nine days before the beginning of pretraining. One $S$ was discarded during training for failure to eat reward pellets, and another $S$ for failure to run in $R 2$. The data of five Ss were discarded to give an $\mathrm{N}$ of eight in each cell.

The double runway used was 3 in. wide and 3-1/2 in. high, and divided by opaque guillotine doors into a 10in. start box (SB), a 16-in. R1, a 14-in. G1, a 39-3/8in. R2, and a 14-in. G2. The first three parts were made of wood and painted flat black; R2 and G2 had wood walls and a floor of 1/2-in. hardware cloth and were painted white. Clear plastic gulllotine doors were mounted behind the opaque doors at SB and between G1 and A2. Jar-lid food cups 2 in. in diameter were positioned near the far ends of G1 and G2. Illumination was provided by two red and one white $7-1 / 2-W$ bulbs located 36 in. above SB, G1, and G2, respectively.

A 1/100-sec clock was started by raising the clear door of SB and stopped by the breaking of a photocell beam $2 \mathrm{in}$. beyond to glve Rl start time; running time was measured from that photocell to a second 12 in. beyond; goal time was measured from the second photo- cell to a third 7 in. from the end of G1. Analogous measures were obtained in R2 with photocells located 2 in. and 14 in. into R2, and 7 in. from the end of G2.

Three days before pretraining half of the Ss (Before groups) received a 3-min test in an open fleld made of a 30-gallon tub painted flat white and illuminated by four 100-W bulbs. The Ss not so treated were the After groups.

On the first pretraining day each $\mathrm{S}$ was handled for $3 \mathrm{~min}$ and exposed to several large (93 $\mathrm{mg}$ ) and small $(45 \mathrm{mg})$ Noyes reward pellets. On the next five days each $S$ was placed in the double runway for 2 min with all doors raised and the timing circuits on. About $30 \mathrm{~min}$ later the $S$ was placed in a box similar to $G 1$ but painted gray, and was exposed to 10 small, 4 large, 5 small, 1 large, and 2 small pellets on each of the five days, respectively.

The Ss assigned to Groups $0,2,4$, or 6 received the indicated number of small pellets in G1 as reward on every trial; all Ss received one large pellet in $\mathbf{G 2}$ on every trial. Trials, given one each day for 21 days, consisted of these steps: (a) S was placed in SB facing R1; (b) the opaque door to $\mathbf{R} 1$ was opened and the clear door opened 1 sec later; (c) the opaque door was lowered as soon as all of $S$ except the tail was past it; (d) as soon as $S$ was past the door to $G 1$ it was closed and the $S$ detained in G1 for $20 \mathrm{sec}$ (e) the doors from G1 to R2 were opened and the $R 1$ procedure repeated, with a 15-sec detection in G2.

Results

Reciprocals of start, running, and goal times for both alleys were determined, and metians of reciprocal times for each block of three trials were used in all analyses. Means of median reciprocal times for $\mathrm{Rl}$ and R2 are plotted in FIg. 1. No breakdown of the BeforeAfter groups was plotted because in the analyses of the six response measures neither the main effects nor any of the interactions of this dimension were gignificant.

In all six analyses $F$ ratios for the effects of trial blocks were significant $(p<.01)$. No other effects were significant for R1 start scores. An effect of reward size on $R 1$ running scores was found $(F=3.24$, df $=$ $3 / 56, p<.05$ ), and analysis by Duncan's multiple range test for $\mathrm{p}<.01$ indicated that homogeneous subsets (in parentheses), in order of decreasing speed, were (Group 6), (Groups 2 and 4), and (Groups 4 and 0 ).

Goal scores for $\mathrm{Rl}$ showed a reward-size effect $(F=$ 4.69, df $=3 / 56, p<.01$ ), and Duncan's range test for $p<.01$ showed homogeneous subsets, in decreasing order, to be (Group 6), (Group 2), and (Groups 4 and 0). The reward by trial-block interaction was significant $(F=2.42, \mathrm{df}=18 / 336, \mathrm{p}<.01)$, and trend analysis showed 


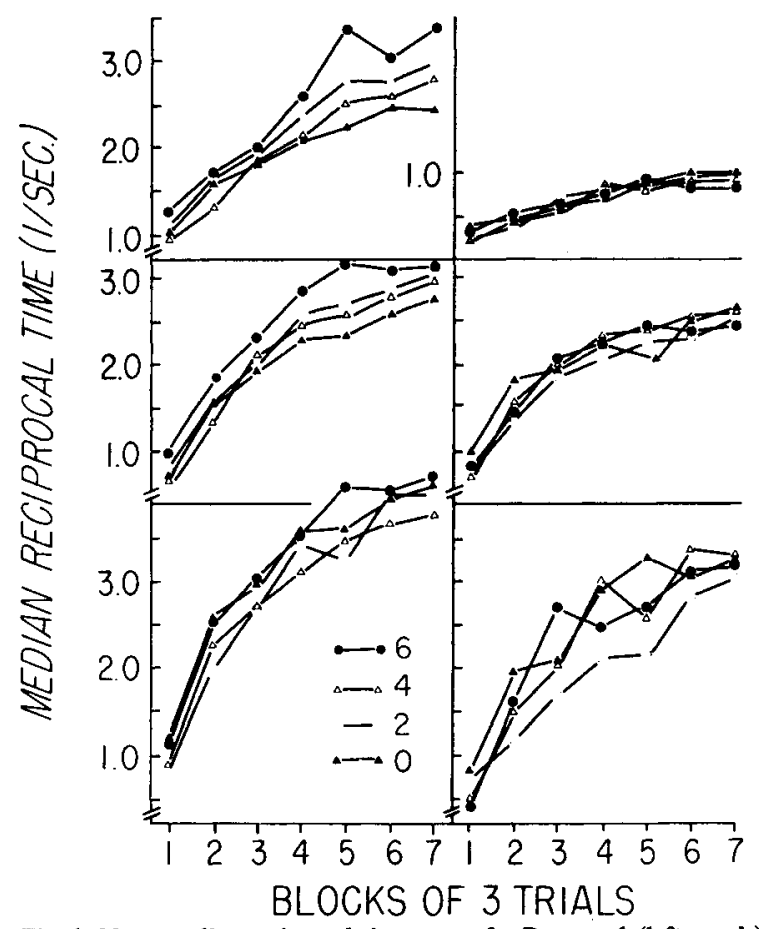

Fig. 1. Mean median reciprocal time scores for Runway-1 (left panels) and Runway-2 (right panels), by blocks of three trials. The panels show start, running, and goal scores from bottom to top.

a significant linear component to this interaction ( $F=$ $7.07, \mathrm{df}=3 / 56, \mathrm{p}<.01$ ). The quadratic and cubic components were not significant, and higher components were not tested.

In $\mathrm{R2}$ the reward by trial-block interaction of start scores was significant $(F=1.67, \mathrm{df}=18 / 336, \mathrm{p}<.05)$, and there was a significant quadratic component to this interaction $(F=4.55, \mathrm{df}=3 / 56, \mathrm{p}<.01)$. No effects other than trials were significant for R2 running and goal scores.

\section{Discussion}

No evidence was obtained for an overall depression of $\mathrm{R2}$ speeds for Groups 2, 4, and 6 compared to Group 0 . The trend analysis of the reward by trial-block interaction for $\mathbf{R 2}$ start scores shows different degrees of bowing of the performance curves for the different reward-size groups. In Fig. 1 this can be seen as less curvature for Group 2 relative to the other groups. Perhaps Ss in this group received just enough reward in G1 to initiate, on early trials, searching in G1 for additional pellets they may have been led to "expect" from pretraining. Upon repeated failure to find more pellets in G1 this behavior extinguishes so that they eventually reach start speeds comparable to the other groups. Such behavior could influence only start scores; R2 running and goal scores are appropriately free of such effects. No searching-for-more-pellets behavior would be elicited for the Group-0 Ss who never received reward in G1, and Ss in Groups 4 and 6 receive sufflcient pellets to "satisfy" reward expectations established during pretraining.

In the Rl data the usual effect of reward size on performance does not appear in the start measure at all, and is clear in the running scores only for the superiority of Group 6. While reliable performance differences are sometimes reported only when differences in reward magnitude are large, this cannot explain Group 2 being intermediate between Groups 6 and 4. Furthermore, the curves for Group 0 are still increasing, i.e., they are acquisition curves, and this is true for all R1 measures. Acquisition of R1 locomotion by Group-0 Ss could be based on secondary reinforcement generalized from G2, but the actual level of performance was little affected by G1 reward magnitude. The rather short narrow runway may have reduced the frequency of competing responses, and the short segments over which responses were timed and the process of averaging over blocks of trials would minimize their influence on the average scores. Thus, the present results support Marx \& Brownstein (1963) since, given the conditions to insure responding, reward size has little effect on the vigor of the response when competing responses are minimal or not scored.

The uniformly nonsignificant effect of emotionality pretesting indicates that pretesting in the open field may be practiced, if desired, without affecting later runway performance. This is fortunate, since the usual indices of emotionality in this test are greatly reduced in occurrence and may show little systematic variation, when the testing is done after training in a runway.

\section{References}

AMSEL, A. The role of frustrative nonreward in noncontinuous reward situations. Psychol Bull, 1958, 55, 102-119.

AMSEL, A., \& ROUSSEL, J. Motivational properties of frustration: $\mathbf{I}$. Effect on a running response of the addition of frustration to the motivational complex. J. exp. PsychoL, 1952, 43, 363-368.

HAMM, H. D. Perseveration and summation of the frustration effect. $J$. exp. Psychol, 1967, 73, 196-203.

MARX, M. H., \& BROWNSTEIN, A. J. Effects of incentive magnitudes on running speeds without competing responses in acquisition and extinction. J. exp. Psychol, 1963, 65, 182-189.

WAGNER, A. R. The role of reinforcement and nonreinforcement in an "apparent frustration effect." J. exp. Psychol, 1959, 57, 130-136. Note

1. Supported by NIH research grants MH08495 and MH12099. The author thanks C. D. Gunn for his assistance. 